\title{
Performance Comparison of CVD Grown Carbon Nanofiber Based on Single- and Multi-Layer Graphene Oxides in Melt-Compounded PA6.6 Nanocomposites
}

\author{
Elcin Cakal Sarac ${ }^{1,2}$, Leila Haghighi Poudeh ${ }^{3}$, Jamal Seyyed Monfared Zanjani ${ }^{4}$, \\ Ilse Letofsky-Papst ${ }^{5}$, Fevzi Çakmak Cebeci ${ }^{6}$, Ismail Aydin'1, Yusuf Menceloglu ${ }^{3,6}$, \\ Burcu Saner Okan ${ }^{3 *}$
}

\author{
${ }^{1}$ Rheology Laboratory, Chemical Engineering Department, Faculty of Engineering, Istanbul University-Cerrahpasa, \\ Avcilar Campus, Avcilar, Istanbul \\ ${ }^{2}$ Kordsa Teknik Tekstil A.S., Istanbul, Turkey \\ ${ }^{3}$ Sabanci University Integrated Manufacturing Technologies Research and Application Center \& Composite Technologies Center \\ of Excellence, Teknopark Istanbul, 34906, Pendik, Istanbul, Turkey \\ ${ }^{4}$ Faculty of Engineering Technology, University of Twente, Enschede, The Netherlands \\ ${ }^{5}$ Institute for Electron Microscopy, Graz University of Technology, Graz, Austria \\ ${ }^{6}$ Faculty of Engineering and Natural Sciences, Materials Science and Nano Engineering, Sabanci University, Istanbul, Turkey \\ Email: *bsanerokan@sabanciuniv.edu
}

How to cite this paper: Sarac, E.C., Poudeh, L.H., Zanjani, J.S.M., Letofsky-Papst, I., Cebeci, F.Ç., Aydin, I., Menceloglu, Y. and Okan, B.S. (2019) Performance Comparison of CVD Grown Carbon Nanofiber Based on Single- and Multi-Layer Graphene Oxides in Melt-Compounded PA6.6 Nanocomposites. Open Journal of Composite Materials, 9, 99-123.

https://doi.org/10.4236/ojcm.2019.92005

Received: February 13, 2019

Accepted: March 29, 2019

Published: April 1, 2019

Copyright (๑) 2019 by author(s) and Scientific Research Publishing Inc. This work is licensed under the Creative Commons Attribution International License (CC BY 4.0).

http://creativecommons.org/licenses/by/4.0/

(c) $\underset{\mathrm{EY}}{\mathrm{C}}$ Open Access

\begin{abstract}
In the present study, newly design hybrid nanostructures were produced by growing long carbon nanofibers (CNF) on single- and multi-layer graphene oxide (GO) sheets in the presence of catalyst by chemical vapor deposition (CVD). Chemical composition analysis indicated the formation of $\mathrm{Fe}-\mathrm{C}$ bonds by the deposition of carbon atoms on catalyst surface of $\mathrm{Fe}_{2} \mathrm{O}_{3}$ and increasing in $\mathrm{C} / \mathrm{O}$ atomic ratio confirming $\mathrm{CNF}$ growing. These hybrid additives were distributed homogeneously through polyamide 6.6 (PA6.6) chains by high shear thermokinetic mixer in melt phase. Spectroscopic studies showed that the differences in the number of graphene layer in hybrid structures directly affected the crystalline behavior and dispersion state in polymer matrix. Flexural strength and flexural modulus of PA6.6 nanocomposites were improved up to $14.7 \%$ and $14 \%$ by the integration of $0.5 \mathrm{wt} \% \mathrm{CNF}$ grown on multi-layer GO, respectively, whereas there was a significant loss in flexural properties of single-layer GO based nanocomposites. Also, the integration of $0.5 \mathrm{wt} \%$ multi-layer GO hybrid reinforcement in PA6.6 provided a significant increase in tensile modulus about $24 \%$. Therefore, multi-layer GO with CNF increased the degree of crystallinity in nanocomposites by forming
\end{abstract}


intercalated structure and acted as a nucleating agent causing the improvement in mechanical properties.

\section{Keywords}

Nanoparticles, Polyamides, Nanocomposites, Mechanical Properties, Thermal Properties

\section{Introduction}

Graphene having a 2-dimensional carbon nanostructure is a promising reinforcing agent in thermoplastic composite materials due to its high mechanical and thermal properties, and high surface area [1] [2]. Several alternative techniques including bottom-up and top-down approaches have been developed in order to synthesize graphene sheets [3]. Chemical vapor deposition (CVD) method is a widely used bottom-up technique to attain defect-free single layer graphene whereas top-down approach includes chemical exfoliation in order to produce large quantities graphene layers in bulk [4] [5] [6]. CVD process provides to grow nanomaterials on metal catalysts or substrates used as a template with gaseous hydrocarbons at elevated temperatures. There are several types of graphene structures used in compounding and polymerization as well as in coating which are graphene oxide (GO), reduced GO, functionalized graphene, expanded graphite or graphene [7] [8]. So far Hummers' method [9] with some modifications and improvements is the most common used route for the synthesis of GO layers. This technique involves the oxidation of graphite by using acidic mixtures and oxidizing agents to exfoliate oxidized graphene layers by breaking down van der Waals forces keeping graphene layers in graphite structure [10]. Also, GO can be exposed to thermal shock that causes the elimination of oxygen groups and form worm-like or vermicular-type structures by the expansion of the crystal lattice of planes in graphite [11] [12]. In thermal treatment, the presence of oxygen functional groups on the surface of GO has great influence on the wettability, hydrophilicity degree, thus providing good compatibility with polymer chains [7] [13]. In addition, GO can be directly reduced by using strong reductants (e.g. hydrazine, hydroquinone, etc.) which remove oxygen containing functional groups.

There have been few attempts to combine the characteristic properties of graphene with the other carbon-based materials such as carbon nanotubes (CNT) or carbon nanofibers (CNF) to develop an hybrid structure by CVD technique [14] [15]. These hybrid structures are mostly preferred in energy storage applications. For instance, Cheng et al. produced $\mathrm{Fe}_{3} \mathrm{O}_{4}$-containing graphene fiber by thermally treating the mixture of GO synthesized by improved Hummers' method and $\mathrm{Fe}_{3} \mathrm{O}_{4}$ nanoparticles in a closed glass pipeline and used these fibers in the construction of flexible supercapacitors [16]. In addition, Li et al. utilized CVD grown CNT on graphene nanoplatelet as a reinforcement in 
epoxy matrix to control load transfer properties and improve the mechanical properties of the composites [17]. At this point, the integration of CNT in hybrid structure prevents the entanglement of nanotubes and thus their agglomeration in polymer matrix [18]. On the other hand, there is still an obstacle in the usage of CNT and graphene separately or together in thermoplastic compounding and their homogeneous dispersion by extrusion process.

There has been a growing interest for thermoplastic composites in the industrial applications due to their low cost, lightweight, recyclable property, and ease of processing at low processing temperature and pressure level [19] [20]. As an important matrix for polymer nanocomposites, polyamide 6.6 (PA6.6), also known as Nylon 6.6, has many different applications in engineering due to high stiffness, toughness, and resistance to dynamic fatigue, high temperature and harsh chemicals [21]. It is possible to produce surface functionalized graphene or CNT based PA nanocomposites with improved mechanical and thermal characteristics by using melt-mixing technique [22] [23]. However, there is no work about the integration of 3D graphene/CNT hybrid additive in thermoplastic polymers in melt phase and the investigation of the effect of graphene type and its number of layers in this hybrid structure with CNF on the performance of PA6.6 nanocomposites.

In the present study, CVD prosess was applied to grow CNF on single- and multi-layer GO templates impregnated by iron catalyst. Detailed structural investigation was implemented in order to find the main differences in these new design hybrids. Later, PA6.6 based nanocomposites reinforced with single-layer GO, multi-layer thermally exfoliated graphene oxide (TEGO), and their CVD grown CNF hybrids were prepared by a thermokinetic high shear mixer to obtain better dispersion and prevent agglomeration of these nano additives. Also, parallel study was conducted with these two types of bicomponent additives to understand the effect of number of graphene layers on the crystallinity and mechanical properties of PA6.6 nanocomposites.

\section{Experimental}

\subsection{Materials}

PA6.6 polymer (Zytel E51HSB NC010) was supplied from Dupont. Thermally exfoliated graphene oxide (TEGO) was purchased from NANOGRAFEN Co. Graphite flakes (+100 mesh size), iron (III) chloride hexahydrate $\left(\mathrm{FeCl}_{3} \cdot 6 \mathrm{H}_{2} \mathrm{O}\right.$, $99 \%)$, dimethylformamide (99\% DMF) and hydrogen peroxide $\left(30 \% \mathrm{H}_{2} \mathrm{O}_{2}\right)$ were purchased from Sigma-Aldrich. Potassium permanganate $\left(\mathrm{KMnO}_{4}\right)$ was purchased from Merck \& Co. Helium (He), hydrogen $\left(\mathrm{H}_{2}\right)$ and ethylene gases were used in CVD experiments.

\subsection{G0 Synthesis}

Graphene oxide (GO) was synthesized from graphite through improved Hummers' method [7] [24]. Briefly, $18 \mathrm{gr}$ of $\mathrm{KMnO}_{4}$ was slowly added to a 9:1 mix- 
ture of concentrated $\mathrm{H}_{2} \mathrm{SO}_{4} / \mathrm{H}_{3} \mathrm{PO}_{4}(360: 40 \mathrm{~mL})$ and $3 \mathrm{gr}$ of graphite flakes. The reaction was then heated up to $50^{\circ} \mathrm{C}$ and stirred for $24 \mathrm{~h}$. The reaction was cooled to room temperature and poured onto ice with $3 \mathrm{~mL} \mathrm{H} \mathrm{H}_{2} \mathrm{O}_{2}$. The mixture was centrifuged at $10,000 \mathrm{rpm}$ for $30 \mathrm{~min}$ to obtain reaction product. The remaining solid material was washed with $200 \mathrm{~mL}$ of distilled water $(\times 2), 200 \mathrm{~mL}$ of $32 \% \mathrm{HCl}(\times 2)$ and $200 \mathrm{~mL}$ of ethanol $(\times 2)$, and the mixture was centrifuged $(10,000 \mathrm{rpm}$ for $30 \mathrm{~min})$ for each washing step. At the final step, the washed material was coagulated with $200 \mathrm{~mL}$ of ether. Then product was kept at $50^{\circ} \mathrm{C}$ under vacuum for 3 days for drying.

\subsection{Iron Impregnation}

Iron $(\mathrm{Fe})$ was used as a catalyst in CVD process to grow CNF on the surface of graphene sheets [25]. Fe impregnation process was applied for two different graphene types in different medium. $0.5 \mathrm{~g} \mathrm{GO}$ was dispersed in $100 \mathrm{ml}$ distilled water for $30 \mathrm{~min}$ by probe sonicator and then $20 \mathrm{~mL}$ of $0.5 \mathrm{M} \mathrm{FeCl}_{3} \cdot 6 \mathrm{H}_{2} \mathrm{O}$ solution was added slowly into GO solution and stirred for $4.5 \mathrm{~h}$ through refluxing. In case of TEGO, $0.5 \mathrm{~g}$ TEGO was dispersed in $100 \mathrm{~mL}$ DMF for $30 \mathrm{~min}$ by probe sonicator to get suspension and homogeneous dispersion, and then $\mathrm{Fe}$ impregnation process was applied similar to that of GO. At the end of each reaction, centrifugation and filtration steps were applied to obtain resultant products. All samples were dried at $70^{\circ} \mathrm{C}$ overnight under vacuum.

\subsection{CNF Growth Process by CVD}

Both graphene types were used for the growth of carbon nanofiber by CVD process to monitor the length of fibers and understand their growing mechanism. Fe loaded graphene samples were placed into a quartz glass plate in the furnace and then $\mathrm{He}$ gas was purged at $50^{\circ} \mathrm{C}$ for 5 min to provide inert atmosphere and prevent oxidization. Subsequently, furnace temperature was increased up to $750^{\circ} \mathrm{C}$ with a heating rate of $35^{\circ} \mathrm{C} /$ min under the mixture of $\mathrm{He}$ and $\mathrm{H}_{2}$ $(3.8: 1=\mathrm{v} / \mathrm{v})$ gases. At $750^{\circ} \mathrm{C}$, temperature and gas flow rates were kept constant for $10 \mathrm{~min}$ for the completion of annealing. Then, ethylene gas was purged to initiate the growth of CNF on graphene templates for $15 \mathrm{~min}$ at $750^{\circ} \mathrm{C}$.

\subsection{Production of PA6.6 Nanocomposites}

GO and TEGO based nano-sized reinforcements were dispersed in PA6.6 matrix at $4700 \mathrm{rpm}$ and $300^{\circ} \mathrm{C}$ by using custom-made Gelimat Thermokinetic Mixer. Both neat graphene and their hybrid contents were adjusted in the range of 0.25 to $1 \mathrm{wt} \%$ to attain ideal dispersion. Then, specimens for mechanical tests were prepared by injection moulding. Two different molds were used in tensile and flexural tests in accordance with ISO 527-2 and ASTM D790 standards, respectively.

\subsection{Characterization}

The morphological structures of the nanoadditives were analyzed by Leo Supra 35VP Field Emission Scanning Electron Microscope (SEM) and JEOL2100 Lab6 
High Resolution Transmission Electron Microscope (TEM). For microtomy analysis, thin sections $(75 \mathrm{~nm})$ were generated with a Cryo-Ultramicrotome (Leica EM UC6/FC6, Leica Microsystems, Vienna, Austria) equipped with an Ultra-Sonic diamond knife (Diatome, Biel, Switzerland) at approximate $-70^{\circ} \mathrm{C}$ and were transferred on a 100 mesh $\mathrm{Cu} / \mathrm{Pd}$ grid (Plano $\mathrm{GmbH}$, Wetzlar, Germany). Despite the cryogenic conditions, the Ultra-Sonic diamond knife generally used at room temperature was used to eliminate compression and attain best structure preservation. The temperature for preparation depends strongly on the sample and the climatic conditions in the room and can only be determined experimentally $\left(-60^{\circ} \mathrm{C}\right.$ to $\left.-80^{\circ} \mathrm{C}\right)$. Thermo Scientific K-Alpha X-ray Photoelectron Spectrometer System (XPS) was used for quantitative elemental analysis of graphene and its hybrid structures. X-ray diffraction (XRD) measurements were carried out by using Bruker D2 PHASER Desktop with a CuK $\alpha$ radiation source. The number of graphene layers was calculated using Debye-Scherrer equation (Equation (1)) below:

$$
\begin{gathered}
t=0.89 \lambda / \beta \cos \theta \\
n=t / d
\end{gathered}
$$

where $t$ is thickness, $\beta$ is full width at half maximum-FWHM and $d$ is interlayer spacing obtained from XRD patterns, and $n$ is the number of graphene layers. Raman spectroscopy was used to identify the structural properties of samples by using a Renishaw in Via Reflex Raman Microscopy System with the laser wavelength of $532 \mathrm{~nm}$ at room temperature in the range of $100-3500 \mathrm{~cm}^{-1}$. Chain conformational study was investigated by Thermo Scientific Fourier Transform Infrared Spectroscopy (FTIR). Thermal characterization was carried out by Mettler Toledo differential scanning calorimeter (DSC) at heating rate of $10^{\circ} \mathrm{C} / \mathrm{min}$ and $300^{\circ} \mathrm{C}$ under $\mathrm{N}_{2}$ atmosphere. The produced compounds were injected by DSM injection molding to prepare tensile and three-point bending test specimens. Mechanical tests were performed with $2 \mathrm{~mm} / \mathrm{min}$ speed by using Instron 5982 Static Test Machine with a $5 \mathrm{kN}$ load cell for tensile (ISO 527-2) and flexural (ASTM D 790) tests of PA6.6 based nanocomposites.

\section{Results and Discussion}

\subsection{Differences in GO and TEGO}

The surface functional groups and number of graphene layers play an important role in the determination of characteristics of graphene based polymeric composites. Especially carbon/oxygen ratio has a significant indicator to understand the dispersion of carbon based additives and improve the interfacial interactions in polymer matrix [26]. TEGO has high carbon content of 97.9 at $\%$ and oxygen content of 2.1 at $\%$ which are obtained from X-ray photoelectron spectroscopy (XPS). TEGO has worm-like structure and Figure 1(a) and Figure 1(b) show the separated graphene layers after thermal expansion that cause the elimination of most of oxygen functional groups from graphene surface. Figure 1(c) and 


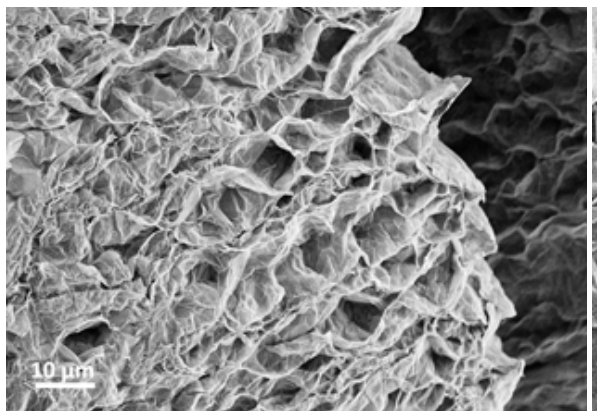

(a)

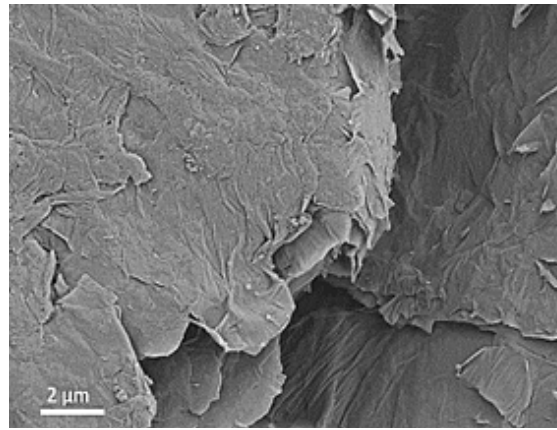

(c)

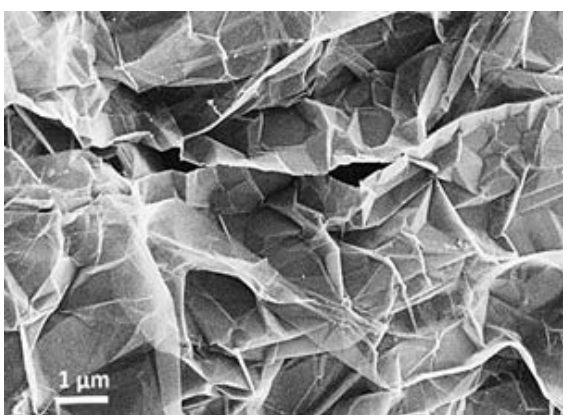

(b)

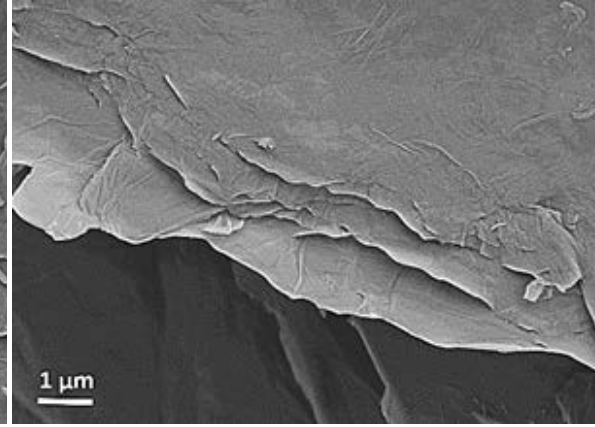

(d)

Figure 1. SEM images of (a-b) TEGO and (c-d) GO sheets at different magnifications.

Figure 1(d) represent GO sheets having smooth and stacked graphene layers. GO contains 60.6 at\% carbon and 34.3 at\% oxygen obtained from XPS measurement and this high amount of oxygen content comes from graphite oxidation process conducted by improved Hummers' method [7].

XRD was used to examine the differences in the structures of TEGO and GO and their XRD spectra are given in Figure 2(a). As seen in XRD spectrum of TEGO, it has characteristic (002) peak at $2 \theta=26.6^{\circ}$ indicating crystalline structure. In addition, the number of graphene layers in TEGO was calculated as 25 , based on (002) characteristic peak of graphene by using Debye-Scherrer equation. On the other hand, GO has a broaden peak at $2 \theta=10^{\circ}$ belonging to (001) peak meaning that it has amorphous structure and graphite flakes were completely oxidized and the intense (002) crystallinity peak of graphitic structure was disappeared as seen in Figure S1(a) representing XRD spectrum of graphite flakes.

Raman characterization technique is widely used as a powerful technique to investigate carbon-based materials by providing useful information about defects and disorders in the structure. In the Raman spectra of graphene, there are three main characteristic peaks at $1355 \mathrm{~cm}^{-1}, 1582 \mathrm{~cm}^{-1}$, and $2721 \mathrm{~cm}^{-1}$ known as $\mathrm{D}, \mathrm{G}$, and $2 \mathrm{D}$ bands, respectively. $\mathrm{D}$ band attributes to the disorderness while $\mathrm{G}$ band corresponds to the vibrational mode of $\mathrm{sp}^{2}$ carbon in graphitic materials and is highly sensitive to the strain changes of $\mathrm{sp}^{2}$ carbons in the structure [27] [28]. As seen in Figure 2(b), TEGO contains three characteristic graphene peaks and its Raman spectrum is also similar to that of bulk graphite since it has 25 
graphene layers graphene. In contrast, GO has D and G bands in which the intensity of $\mathrm{D}$ band considerably increased due to the formation of defects in the oxidation process [29]. Raman spectrum of graphite flakes is provided in Figure S1(b) in supplementary document. These results confirmed that the number of graphene layer was tailored by changing chemistry routes.

\subsection{Comparative Study of Growing Behavior of CNF-GO and CNF-TEGO}

The growth mechanism of CNF is directly affected by the alterations in C/O ratio and the type of the template used in CVD process. In order to understand the deposition of carbon atoms on catalyst surface and control the surface chemistry, binding energies and surface compositions of CNF grown on TEGO and GO were defined by XPS analysis. The intensities of C1s and O1s of GO, TEGO and their hybrid structures were compared in the XPS survey scan spectra in Figure 3(a). Elemental analysis clarified the significant increase in $\mathrm{C} / \mathrm{O}$ atomic ratio from 3.4 of TEGO up to 6.9 of CNF-TEGO confirming the deoxygenation and CNF formation on the surface of TEGO during CVD process. In case of GO, after CVD growth process, carbon content of GO has dramatically increased from 60.6 up to 96.8 at\% indicating the formation of CNF in the structure. Also, $\mathrm{C} / \mathrm{O}$ ratio of CNF-GO became 5.5 whereas that of neat GO was estimated as 0.5 . Similar to CNF-TEGO, CNF were grown randomly on the surface of single-layer GO after CVD process. After CVD process, CNF were grown randomly on the surface of TEGO while the content of carbon increased slightly up to 98.1 at $\%$. Table S1 summarizes elemental composition and C/O ratio of neat and hybrid samples.

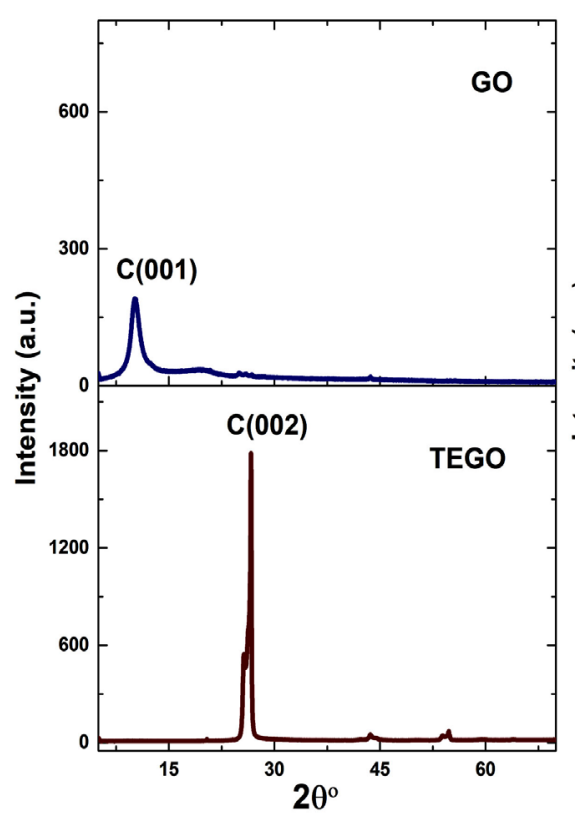

(a)

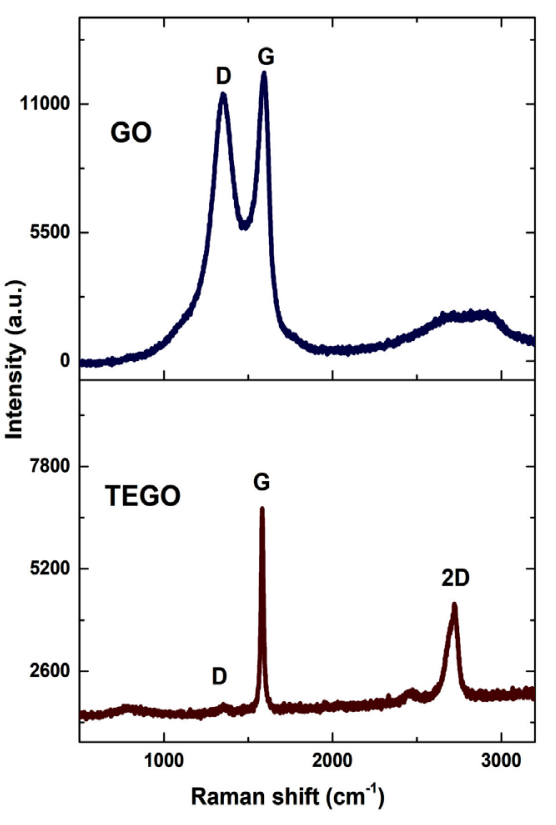

(b)

Figure 2. (a) XRD spectra and (b) Raman spectra of GO and TEGO. 


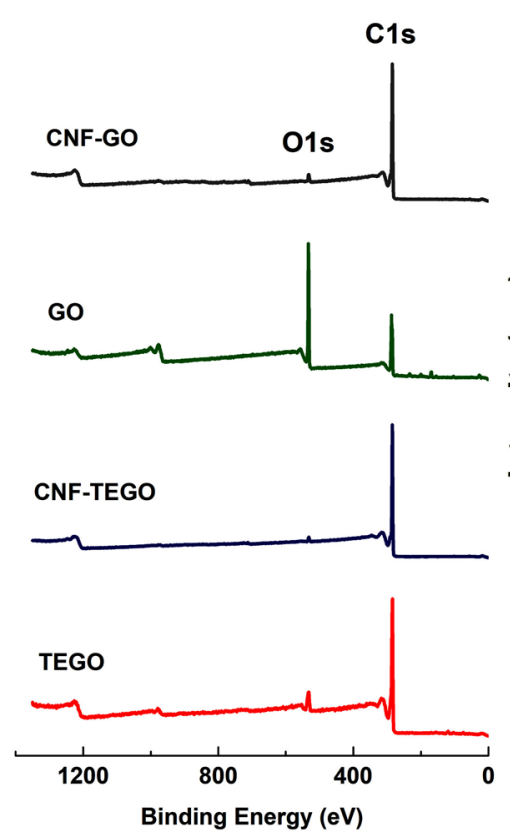

(a)

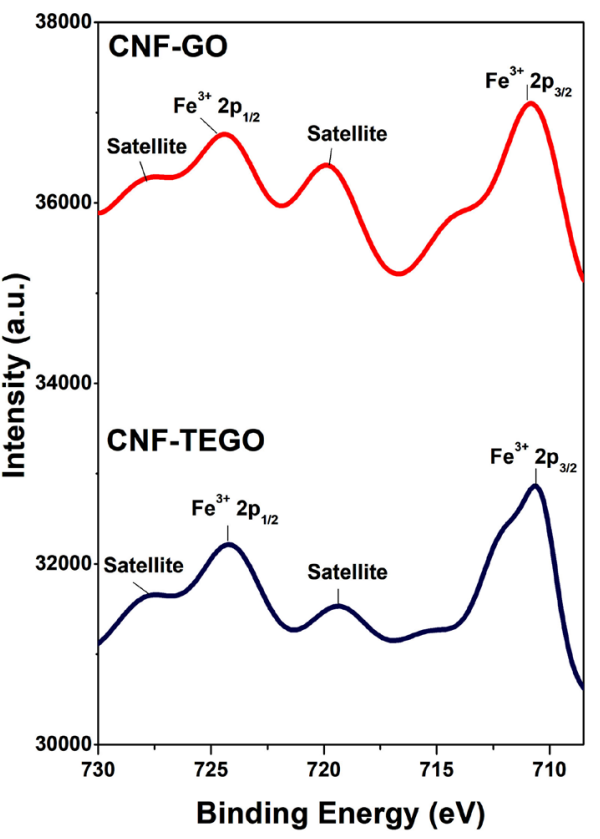

(b)

Figure 3. (a) XPS survey scan spectra of TEGO, CNF-TEGO, GO, and CNF-GO and (b) Fe2p of CNF-TEGO and CNF-GO.

In addition to $\mathrm{C}$ and $\mathrm{O}$ analysis, the $\mathrm{Fe} 2 \mathrm{p}$ envelope of $\mathrm{GO}$ and TEGO hybrid additives provided information about the ionic bond character of the iron. In Figure 3(b), the peaks of $\mathrm{Fe} 2 \mathrm{p}_{1 / 2}$ and $\mathrm{Fe} 2 \mathrm{p}_{3 / 2}$ in CNF-TEGO based on the ionic states of Fe are located at around $724.2 \mathrm{eV}$ and $710.6 \mathrm{eV}$, respectively. CNF-GO also contained similar two peaks at $724.5 \mathrm{eV}$ and $710.7 \mathrm{eV}$. Furthermore, these two main peaks have satellite peaks indicating the presence of $\mathrm{Fe}_{2} \mathrm{O}_{3}$ on the surface of graphene layers confirmed by XRD [30]. The $\mathrm{C} 1 \mathrm{~s}$ and O1s envelopes consist of $\mathrm{Fe}-\mathrm{C}$ and $\mathrm{Fe}-\mathrm{O}$ bonds that supported chemical binding between graphene and CNF in the presence of iron catalyst. The detailed information about the type of bonding in C1s, O1s and Fe2p scan survey is given in Table S2.

Macroscopic studies by TEM and SEM were also conducted to observe the length and diameter of CNF in TEGO and GO. Multi-layer TEGO based hybrid additive has randomly distributed carbon fibers as seen in Figure 4(a). The average length of CNF grown on TEGO is about $2 \mu \mathrm{m}$ and its diameter is measured as $28 \mathrm{~nm}$ in Figure 4(c) and its inset figure. On the other hand, single-layer GO based structure has randomly oriented CNF in its structure as shown in Figure 4(b). The length and diameter of CNF grown on single-layer GO are approximately $1.6 \mu \mathrm{m}$ and $34 \mathrm{~nm}$ in Figure 4(d) and its inset.

The structural changes of hybrid structures of TEGO and GO were monitored by XRD analysis. In Figure 5(a), neat TEGO has sharp (002) graphitic plane peak. After CVD process, the intensity of (002) peak significantly decreased due to the randomly deposited carbon atoms and thus the reduction in crystallinity. In contrast, GO has (001) amorphous peak but growing carbon fiber on GO surface caused the formation of crystalline structure lower than TEGO-based 
material and also (002) peak appeared as seen in Figure 5(b). In addition to characteristic peaks of GO and TEGO, the additional peaks at $2 \theta=42.8^{\circ}$ and $43.6^{\circ}$ in the XRD spectra of both CNF-TEGO and CNF-GO are associated with super-positions of (100) and (101) reflections of the graphite structure [31]. During CNF growth process, iron oxide catalyst was reduced to metallic $\mathrm{Fe}$ and $\mathrm{Fe}_{3} \mathrm{C}$ [32]. Therefore, the characteristic peaks at $2 \theta=37.6^{\circ}$ and $44.9^{\circ}$ are attributed to (311) and (400) planes of Fe catalyst [33], and reflections at $2 \theta=45.7^{\circ}$, $48.4^{\circ}$, and $49^{\circ}$ are corresponding to (112), (131), and (221) planes of orthorhombic $\mathrm{Fe}_{3} \mathrm{C}$ phase, respectively [34]. Therefore, XRD confirmed the deposition of carbon atoms on iron metal and thus the growth of CNF on graphene templates.

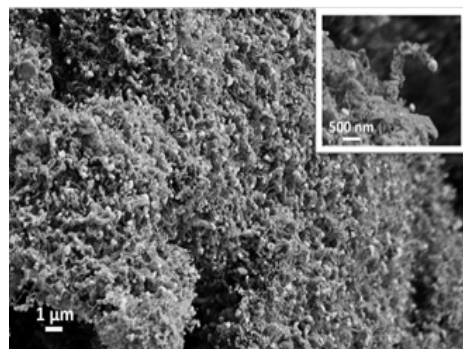

(a)

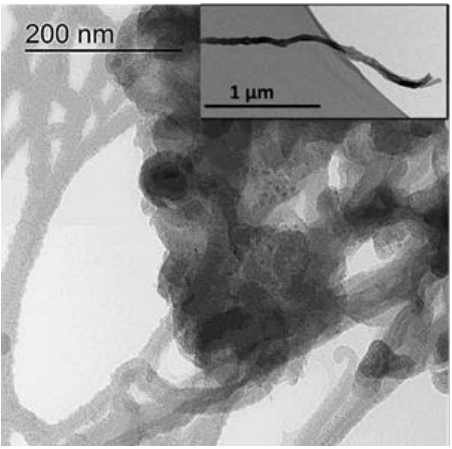

(c)

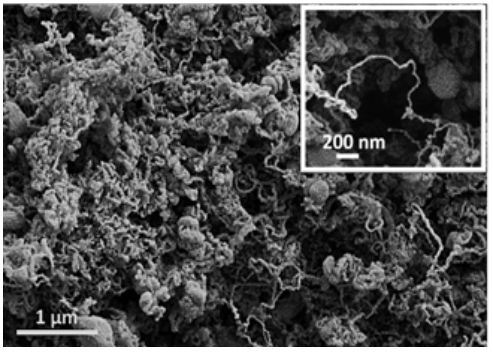

(b)

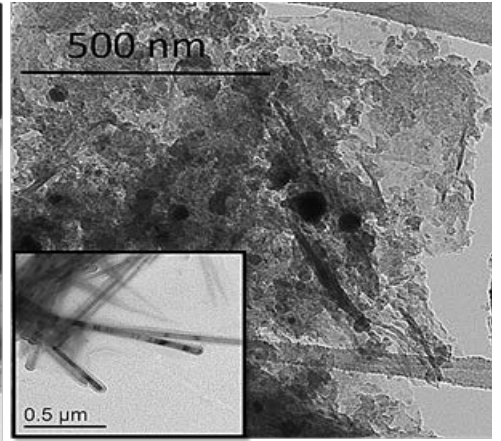

(d)

Figure 4. SEM images of CNF grown on (a) TEGO and (b) GO, and TEM images of CNF grown on (c) TEGO and (d) GO.

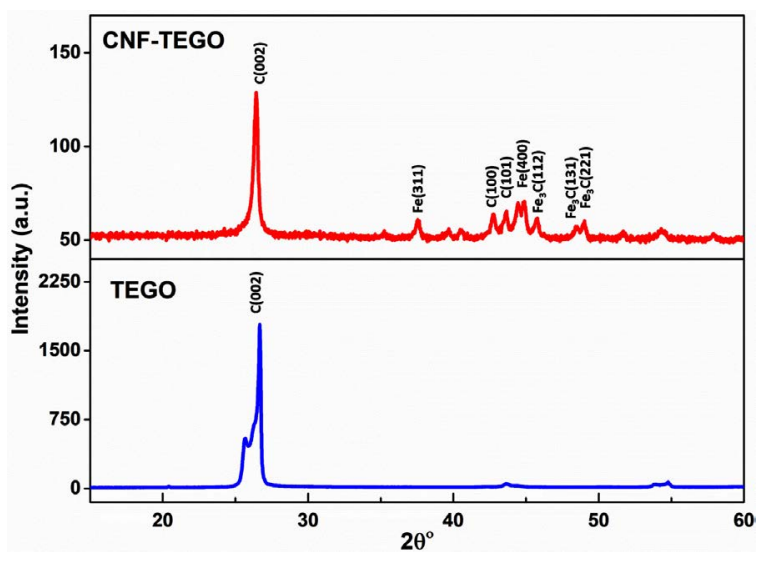

(a) 


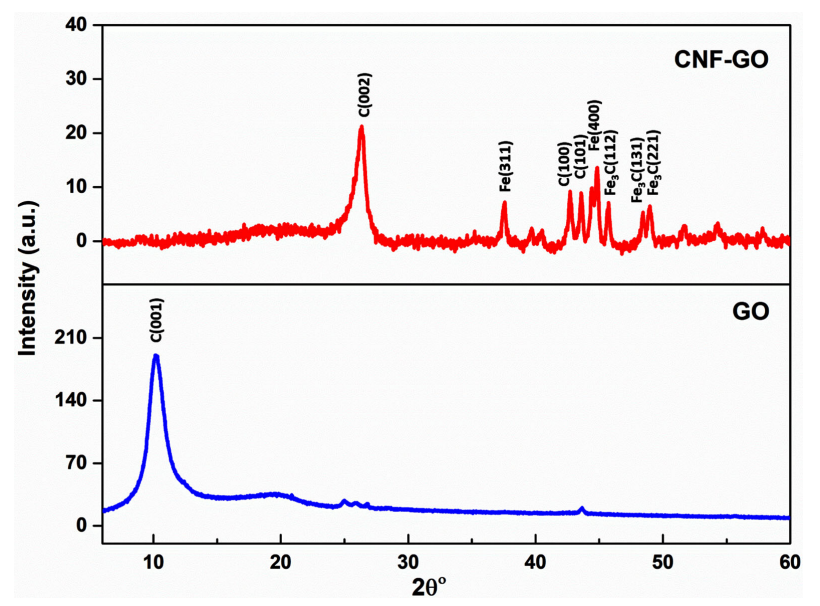

(b)

Figure 5. XRD spectra of (a) TEGO and CNF-TEGO, and (b) GO and CNF-GO.

Spectral analysis by Raman scattering was also conducted to understand the alterations in the new structures. Figure 6 shows the comparison of Raman peaks of neat TEGO and GO and their CVD grown CNF hybrid samples. After CVD process, a shoulder in the G band of both CNF-GO and CNF-TEGO shown in the insets of Figure 6 appeared as a result of change in the geometry of the samples during the growth of tube like CNF [27]. In addition, a second-order peak known as S3 at around $2929 \mathrm{~cm}^{-1}$ in the Raman spectra of both CNF-TEGO and CNF-GO was relied on intervalley and intravalley phonon scattering between graphene and CNF [35] [36]. Furthermore, the formation of CNF affected the disorderness and crystallinity of the structure and significant changes was observed in the intensity ratio of $D$ to $G$ peaks $\left(I_{D} / I_{G}\right)$ that increased by increasing the size of the crystalline grains or interdefect distance [37]. Neat TEGO has very small $\mathrm{D}$ peak whereas a sharp increase in the intensity of $\mathrm{D}$ band is observed after CVD process. GO also has similar trend and $\mathrm{I}_{\mathrm{D}} / \mathrm{I}_{\mathrm{G}}$ of its hybrid increases up to 1.5 from after CVD process. Table S3 summarizes peak positions and $\mathrm{I}_{\mathrm{D}} / \mathrm{I}_{\mathrm{G}}$ values of neat and hybrid structures.

\subsection{Comparative Study of CNF-TEGO and CNF-GO Reinforced PA6.6 Nanocomposites}

The changes in crystallinity and dispersion state of neat PA6.6 and its nanocomposites were investigated via XRD technique and the results are given in Figure $7(\mathrm{a})$ and Figure $7(\mathrm{~b})$. The prominent (100) peak at around $2 \theta=21^{\circ}$ corresponds to $\alpha$-crystalline phase of PA6.6 due to intrasheet scattering within polymeric chains [38]. After the integration of nanofillers into PA6.6 matrix, two new peaks at $2 \theta=11^{\circ}$ and $23^{\circ}$ were appeared in the XRD spectrum of nanocomposites and attribute to the (002) reflection of $\gamma$ phase and $(010 / 110)$ reflection of $\alpha$-crystal structure, respectively [39] [40]. In contrast to the (100) reflection peak, (010/110) peak belongs to the intersheets scattering of hydrogen-bonded PA6.6 sheets [38]. Moreover, the incorporation of nanofiller promotes the $\gamma$ 
phase by inhibiting the chain mobility to form intercalated structure and thus limiting the formation of hydrogen-bonded sheets and $\alpha$ crystalline structure [28] [41]. The current study demonstrated that the layer number had a significant influence on polymer crystallization behavior and multi-layered structure of TEGO based additive led to an increase in crystallinity due to the creation of percolated domains in the polymer matrix. In other words, TEGO has prominent (002) peak attributed to crystallinity and graphite based structure (Figure 5(a)) since it has 25 layers keeping each other by van der Waals interactions and thus TEGO based hybrid additive caused the formation of intercalated structure by the coverage of polymer chains. Contrarily, the intensity of (010/110) peak slightly decreased in CNF grown on GO based nanocomposite and it has more intense $\gamma(002)$ peak and shifting of this peak is higher at around $2 \theta=10^{\circ}$ than TEGO based one (Figure 7(b)). This means that GO based hybrid additive resulted in intercalated structure since GO has broad (001) peak due to its single-layer and amorphous structure (Figure 5(b)) and its hybrid has comparably low (002) intensity peak owing to the growth of CNF.

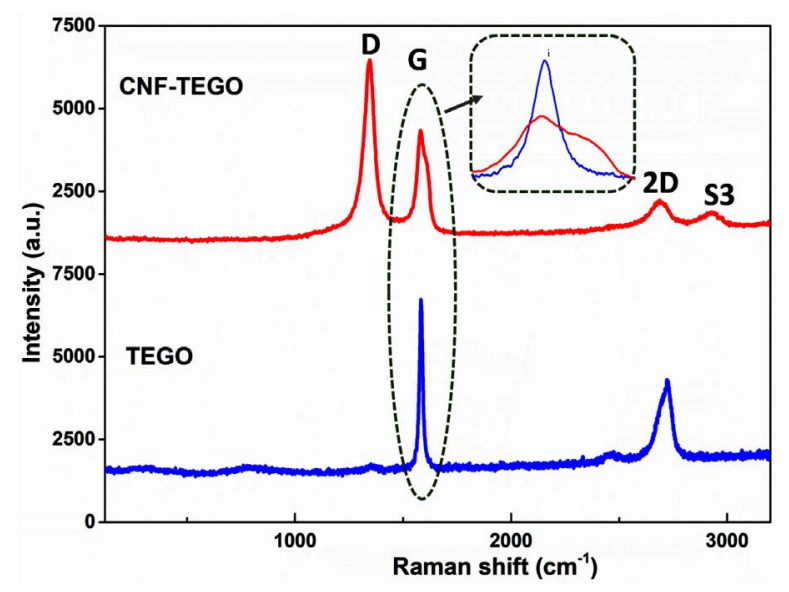

(a)

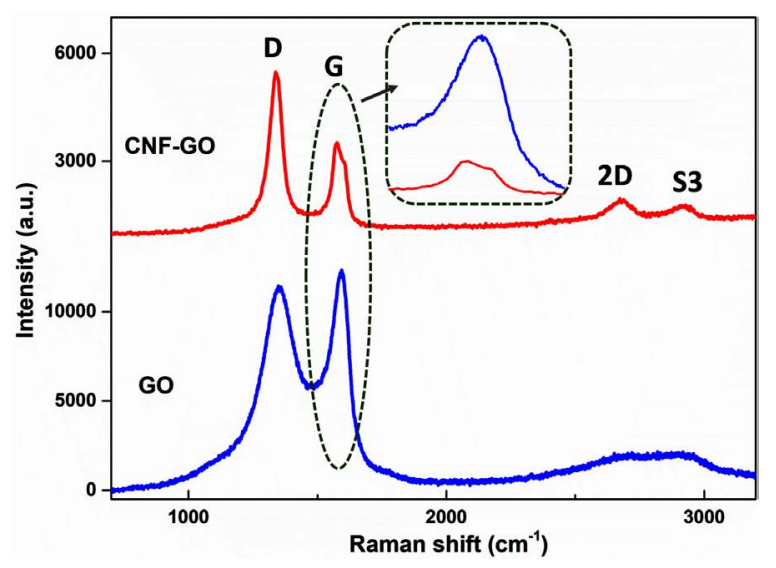

(b)

Figure 6. Raman spectra of (a) TEGO and CNF-TEGO, and (b) GO and CNF-GO. 


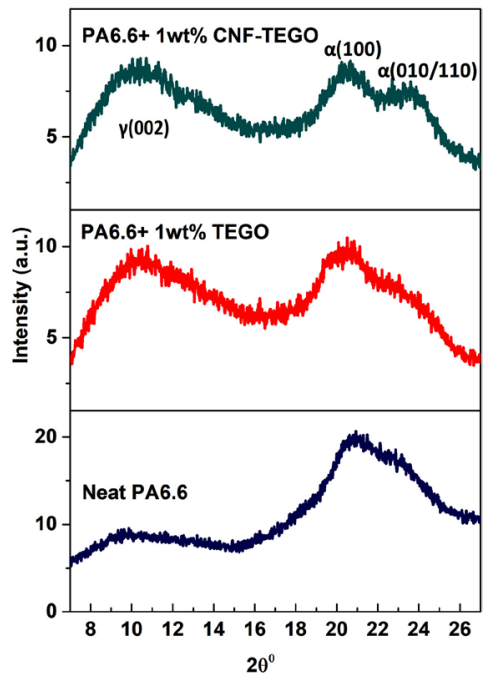

(a)

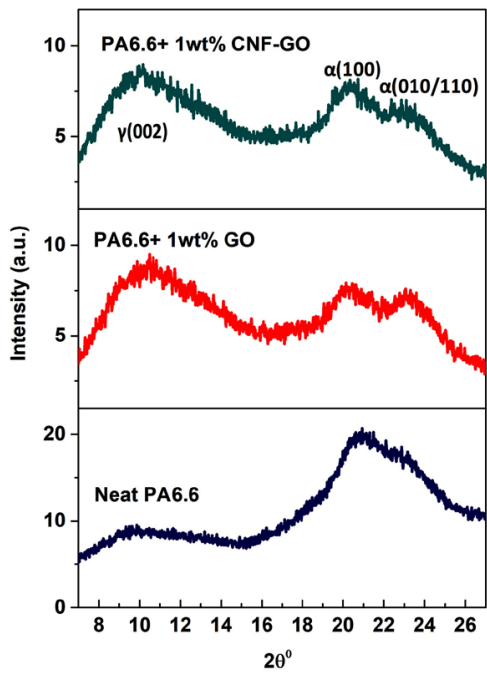

(b)

Figure 7. XRD spectra of PA6.6 composites having (a) 1 wt $\%$ TEGO and 1 wt\% CNF-TEGO, and (b) $1 \mathrm{wt} \%$ GO and $1 \mathrm{wt} \%$ CNF-GO.

FTIR analysis was also conducted to monitor the influence of hybrid additives on the crystallinity of PA6.6 nanocomposites. In Figure 8(a) and Figure 8(b), neat PA6.6 and $1 \mathrm{wt} \%$ TEGO-CNF and $1 \mathrm{wt} \%$ CNF-GO reinforced nanocomposites exhibited two crystalline bands at $934 \mathrm{~cm}^{-1}$ and $1198 \mathrm{~cm}^{-1}$ [42] [43], and the amorphous peak at $1180 \mathrm{~cm}^{-1}$ [44]. Sharp peak at $1630 \mathrm{~cm}^{-1}$ is independent of crystallinity and attributed to the amide $\mathrm{I}, \mathrm{C}=\mathrm{O}$ streching vibration [42]. In this study, the peak at $1630 \mathrm{~cm}^{-1}$ was used as a reference band in order to understand the changes in crystallinity [45]. In Table 1 and Table S4, the transmittance ratios of $\left(\mathrm{T}_{935} / \mathrm{T}_{1630}\right)$ and $\left(\mathrm{T}_{1198} / \mathrm{T}_{1630}\right)$ represent the changes in crystallinity structure of nanocomposites and the results are complementary with each other. The embedment of CNF-TEGO and CNF-GO into the nanocomposites enhanced the crystallinity. The results designated that graphene based bicomponent fillers act as an effective nucleating agent comparably better than neat PA6.6 polymer.

DSC technique was employed to investigate the crystalline behavior of nanocomposites and obtain a deep understanding of nucleation effect of hybrid additives. Figure 9 exhibits DSC curves of neat PA6.6 and 1 wt\% hybrid reinforced PA6.6 nanocomposites. It was found that there is no big difference in melting temperature $\left(\mathrm{T}_{\mathrm{m}}\right)$ of nanocomposites specimens when compared to neat PA6.6 polymer according to Table 2. The percent crystallinity is determined using Equation (2):

$$
X_{c}=\left(\Delta H_{m} / \Delta H_{m}{ }^{100 \%}\right) \times 100 \%
$$

where $X_{c}$ is the degree of crystallinity, $\Delta H_{m}$ is enthalpy of melting, and $\Delta H_{m}{ }^{100 \%}$ is enthalpy of purely crystalline $(\mathrm{J} / \mathrm{g}) . \Delta H_{m}{ }^{100 \%}$ is a reference value for purely crystalline PA6.6 as about $188.4 \mathrm{~J} / \mathrm{g}$ [43]. The $\Delta H_{m}$ of neat PA6.6 at the melting temperature was calculated as $48.2 \mathrm{~J} / \mathrm{gr}$. In Table 2, there was a sharp increase in $X_{c}$ values by the addition of each type of graphene hybrid additive. These results 
confirmed that graphene based additives were acted as effective nucleating agents by allowing crystal growth during crystallization. However, we could not detect any significant difference between single- and multi-layer based structures in bulk matrix. Therefore, mechanical behavior of the produced nanocomposites is a key indicator to point out crystallization and the detailed explanation is given in following section.

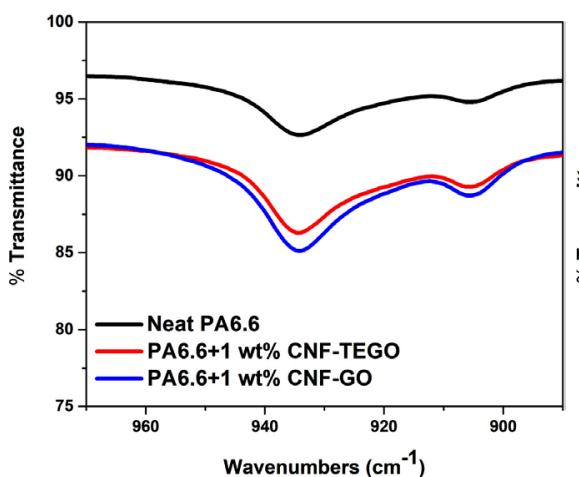

(a)

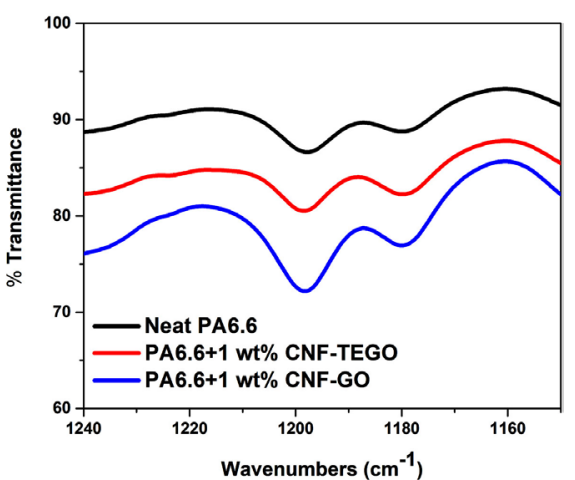

(b)

Figure 8. FTIR spectra of (a) crystalline band and amide axial deformation $(\mathrm{C}-\mathrm{C}=\mathrm{O})$ of neat PA6.6 and $1 \mathrm{wt} \%$ CNF-TEGO and CNF-GO loaded nanocomposites, (b) crystalline band and symmetrical angular deformation out of plane and (c) the amide I, $\mathrm{C}=\mathrm{O}$ stretching vibration of neat PA6.6 and 1 wt\% CNF-TEGO and CNF-GO loaded nanocomposites.

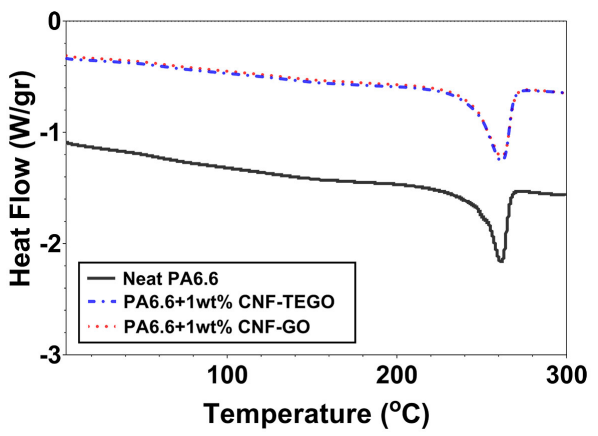

Figure 9. DSC curves of neat PA6.6 and PA6.6 nanocomposites having $1 \mathrm{wt} \%$ CNF-TEGO and CNF-GO.

Table 1. The transmittance ratio of crystalline and independent peaks $\left(\mathrm{T}_{935 / 1630}\right)$.

\begin{tabular}{cccc}
\hline Samples & $\mathrm{T}_{935}$ & $\mathrm{~T}_{1630}$ & $\mathrm{~T}_{935 / 1630}$ \\
\hline Neat PA6.6 & 92.6 & 72.4 & 1.28 \\
PA6.6 + 0.25 wt\% CNF-TEGO & 83.6 & 42.0 & 1.99 \\
PA6.6 + 0.5 wt\% CNF-TEGO & 82.0 & 36.3 & 2.26 \\
PA6.6 + 1 wt\% CNF-TEGO & 86.3 & 55.3 & 1.56 \\
PA6.6 + 0.25 wt\% CNF-GO & 86.1 & 33.7 & 2.55 \\
PA6.6 + 0.5 wt\% CNF-GO & 85.8 & 29.5 & 2.91 \\
PA6.6 + 1 wt\% CNF-GO & 85.1 & 44.6 & 1.91 \\
\hline
\end{tabular}


Table 2. DSC results of CNF-TEGO and CNF-GO reinforced nanocomposites with different filler amounts.

\begin{tabular}{|c|c|c|c|}
\hline Samples & $\mathrm{T}_{\mathrm{m}}\left({ }^{\circ} \mathrm{C}\right)$ & $\Delta H_{m}(\mathrm{~J} / \mathrm{gr})$ & $X_{c}(\%)$ \\
\hline Neat PA6.6 & 262 & 48.2 & 25.6 \\
\hline $\begin{array}{c}\text { PA6.6 + } 0.25 \mathrm{wt} \% \\
\text { CNF-TEGO }\end{array}$ & 262 & 77.4 & 41.1 \\
\hline $\begin{array}{c}\text { PA6. } 6+0.5 \text { wt } \% \\
\text { CNF-TEGO }\end{array}$ & 262 & 74.2 & 39.4 \\
\hline $\begin{array}{c}\text { PA6.6 + } 1 \mathrm{wt} \% \\
\text { CNF-TEGO }\end{array}$ & 262 & 75.4 & 40.0 \\
\hline $\begin{array}{c}\text { PA6. } 6+0.25 \mathrm{wt} \% \\
\text { CNF-GO }\end{array}$ & 261 & 73.4 & 39.0 \\
\hline $\begin{array}{c}\text { PA } 6.6+0.5 \mathrm{wt} \% \\
\text { CNF-GO }\end{array}$ & 262 & 74.2 & 39.4 \\
\hline $\begin{array}{c}\text { PA6.6 + } 1 \mathrm{wt} \% \\
\text { CNF-GO }\end{array}$ & 261 & 73.9 & 39.2 \\
\hline
\end{tabular}

\subsection{Mechanical Behavior of CNF-TEGO and CNF-GO Reinforced Composites}

After the fabrication of single- and multi-layer graphene based hybrid reinforcements, these two fillers were dispersed at the loadings of $0.25,0.5$ and 1 wt\% in PA6.6 matrix by a thermokinetic mixer. Typical stress-strain curves of neat PA6.6 and its nanocomposites of tensile and three-point bending tests are shown in Figure 10. The tensile stress-strain curves exhibited a linear initial response to the applied load (strain values under $0.01 \mathrm{~mm} / \mathrm{mm}$ ) for both reinforcement types in Figure 10(a) and Figure 10(b). Tensile modulus calculated in the linear regions between 0.0005 and $0.0025 \mathrm{~mm} / \mathrm{mm}$ indicated that the modulus of PA6.6 was enhanced up to $24 \%$ by the incorporation of $0.5 \mathrm{wt} \%$ CNF-TEGO in the polymer structure whereas increasing CNF-TEGO amount to $1 \mathrm{wt} \%$ decreased the modulus improvement down to $17 \%$ compared to neat PA6.6 specimen. On the other hand, the incorporation of only $0.25 \mathrm{wt} \% \mathrm{CNF}-\mathrm{GO}$ improved the tensile modulus by $34 \%$ while $0.5 \mathrm{wt} \%$ and $1 \mathrm{wt} \%$ CNF-GO specimens showed $20 \%$ and $30 \%$ enhancement in the tensile modulus, respectively. Table 3 summarizes the tensile properties of the PA6.6 and its nanocomposites in terms of modulus and strength and their improvement values in comparison of neat specimen. The variation in tensile modulus stems from non-uniformity in dispersion state and the effect of nano reinforcement on the crystal structure of the composites. However, increasing the reinforcement amount lowered the dispersion quality and caused the agglomeration of particles in the composite structure and thus decreased modulus values. Furthermore, tensile strength showed improvement in all cases of nanocomposites compared to neat PA6.6 specimen except for PA6.6 + 1 wt\% CNF-GO in which tensile strength was dropped by $7.7 \%$ associated with the lower chemical compatibility of CNF-GO with PA6.6 and the presence of defects in the composite structure. Specimens 
reinforced by CNF-TEGO showed higher tensile strength compared to CNF-GO ones indicating higher interfacial interaction between CNF-TEGO and PA6.6 and slightly higher crystallinity in these specimens compared to specimens reinforced by CNF-GO confirmed by DSC characterization. Also, XRD characterization supported that CNF-TEGO has higher crystallinity than CNF-GO discussed in Section 3.2. The highest strength value was achieved with PA6.6 + $0.5 \mathrm{wt} \%$ CNF-TEGO specimen as about $80.6 \mathrm{MPa}$ which is $15.5 \%$ higher than neat PA6.6. Moreover, the higher decrease in the strain at failure of specimens reinforced by CNF-GO compared to CNF-TEGO ones was due to lower compatibility of CNF-GO with PA6.6 and its chemical composition obtained from XPS characterization. Figures 10 (c) and Figures 10(d) represent the flexural stress and strain curves of CNF-TEGO and CNF-GO reinforced specimens, respectively. Table 4 indicated that the flexural properties of CNF-TEGO reinforced PA6.6 nanocomposites were higher than those of CNF-GO reinforced nanocomposites. The best flexural property was achieved by using $0.5 \mathrm{wt} \%$ CNF-TEGO providing $14 \%$ improvement in flexural modulus and $14.7 \%$ in flexural strength. Consequently, CNF grown on multi-layer GO at $0.5 \mathrm{wt} \%$ loading showed better performance in both tensile and flexural tests since the diameter of fibers on multi-layer GO is smaller than the one grown on single-layer GO as depicted in Figure 4 and this hybrid might reduce stress concentration sites in the matrix by enlarging the specific surface area in the matrix at optimum loading. In addition, crystallinity degree of nanoadditive is a crucial factor to attain better mechanical performance and multi-layer TEGO based additive showed slightly higher crystallinity and thus improved mechanical properties.

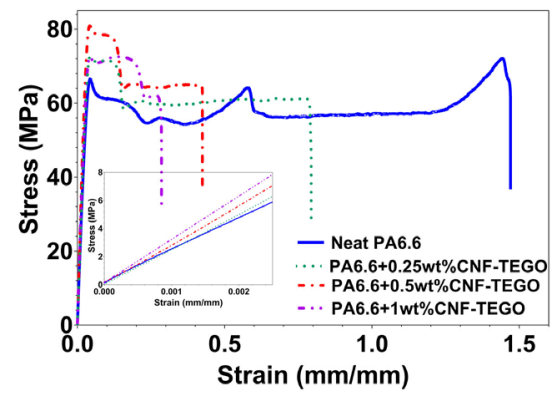

(a)

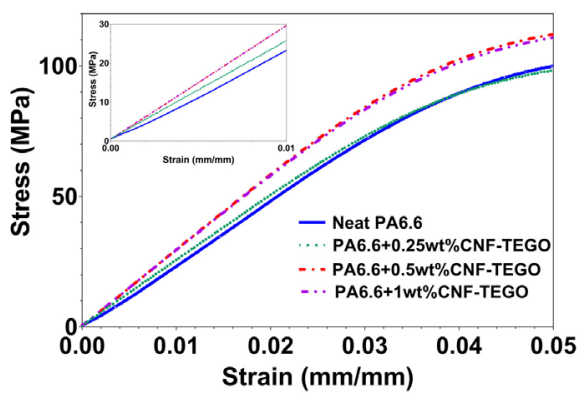

(c)

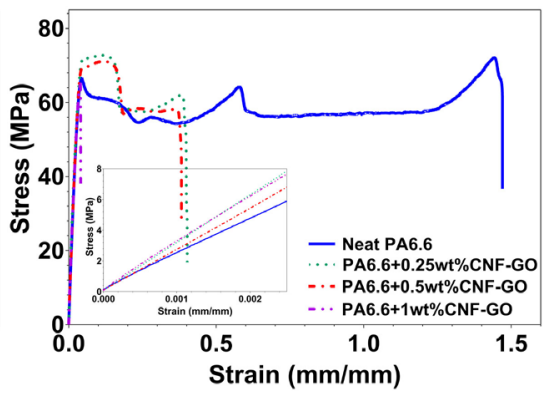

(b)

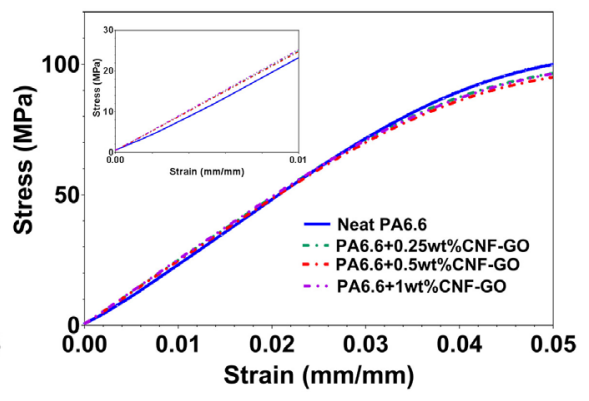

(d)

Figure 10. (a, b) Tensile stress-strain curves and (c, d) Flexural stress-strain curves of CNF-TEGO and CNF-GO based nanocomposites at different loading ratios. 
Table 3. Summary of tensile properties of PA6.6 + CNF-TEGO and PA6.6 + CNF-GO nanocomposites.

\begin{tabular}{ccccc}
\hline Samples & $\begin{array}{c}\text { Tensile } \\
\text { Modulus } \\
(\mathrm{MPa})\end{array}$ & $\begin{array}{c}\text { Tensile Modulus } \\
\text { Improvement } \\
(\%)\end{array}$ & $\begin{array}{c}\text { Tensile } \\
\text { Strength } \\
(\mathrm{MPa})\end{array}$ & $\begin{array}{c}\text { Tensile Strength } \\
\text { Improvement } \\
(\%)\end{array}$ \\
\hline Neat PA6.6 & 2400 & - & 69.8 & - \\
$\begin{array}{c}\text { PA6.6 + 0.25 wt\% } \\
\text { CNF-TEGO }\end{array}$ & 2586 & 8 & 71.8 & 2.9 \\
PA6.6 + 0.5 wt\% & 2971 & 24 & 80.6 & 15.5 \\
CNF-TEGO & 2811 & 17 & 74.8 & 7.2 \\
PA6.6 + 1 wt\% CNF-TEGO & 3225 & 34 & 72.1 & 3.3 \\
PA6.6 + 0.25 wt\% CNF-GO & 32256.7 & 2.7 \\
PA6.6 + 0.5 wt\% CNF-GO & 2868 & 20 & 71.7 & -7.7 \\
PA6.6 + 1 wt\% CNF-GO & 3122 & 30 & 64.4 & \\
\hline
\end{tabular}

Table 4. Summary of flexural properties of PA6.6 + CNF-TEGO and PA6.6 + CNF-GO nanocomposites.

\begin{tabular}{ccccc}
\hline Samples & $\begin{array}{c}\text { Chord } \\
\text { Modulus } \\
(\mathrm{MPa})\end{array}$ & $\begin{array}{c}\text { Flexural Modulus } \\
\text { Improvement (\%) }\end{array}$ & $\begin{array}{c}\text { Flexural } \\
\text { Strength } \\
(\mathrm{MPa})\end{array}$ & $\begin{array}{c}\text { Flexural Strength } \\
\text { Improvement } \\
(\%)\end{array}$ \\
\hline $\begin{array}{c}\text { Neat PA6.6 } \\
\text { PA6.6 + 0.25 wt\% } \\
\text { CNF-TEGO }\end{array}$ & 2570 & - & 102 & - \\
PA6.6 + 0.5 wt\% CNF-TEGO & 2935 & -1 & 103 & 1.0 \\
PA6.6 + 1 wt\% CNF-TEGO & 2904 & 14 & 117 & 14.7 \\
PA6.6 + 0.25 wt\% CNF-GO & 2485 & 13 & 116 & 13.7 \\
PA6.6 + 0.5 wt\% CNF-GO & 2365 & -3 & 101 & -1.0 \\
PA6.6 + 1 wt\% CNF-GO & 2480 & -8 & 98 & -3.9 \\
\hline
\end{tabular}

\subsection{Cross-Sectional Analysis of CNF-TEGO and CNF-GO Hybrid Reinforced PA6.6 Composites}

Cross-sectional study was held for both fracture surfaces and cutting edges of the produced nanocomposites. Freezed fracture surfaces of neat PA6.6 and its nanocomposite specimens with $1 \mathrm{wt} \%$ loading of CNF-TEGO and CNF-GO were examined by SEM technique. Neat PA6.6 has fragmented surface in Figure 11(a). The addition of CNF-TEGO in the host matrix reduces surface roughness compared to neat PA6.6, as shown in Figure 11(b). In case of CNF-GO in Figure 11(c), surface is comparably smoother than both neat PA6.6 and CNF-TEGO based nanocomposite. This might come from GO having flattened surface whereas TEGO has worm-like structure and expanded layers as seen in Figure 1.

In order to provide better understanding in the dispersion of nanoadditives in polymer matrix, microtomy analysis from the center of each specimen in a vertical alignment was carried out. Figure 12(a) and Figure 12(b) represent mi- 
crotome surfaces of $1 \mathrm{wt} \%$ CNF-TEGO reinforced PA6.6 nanocomposites having distributed broken long fibers and separated graphene sheets since an applied high shear rate during melt compounding can cause to break down long CNF from TEGO surface. On the otherhand, TEM images of 1wt\% CNF-GO based PA6.6 specimens have broken fibers shown in Figure 12(c) and clustering of wrinkled GO sheets as seen in Figure 12(d). Consequently, the results are supported the mechanical tests mentioned in the Section 3.4 and TEGO based hybrid additive has better performance than CNF-GO based composites due to its good dispersion quality in host matrix.

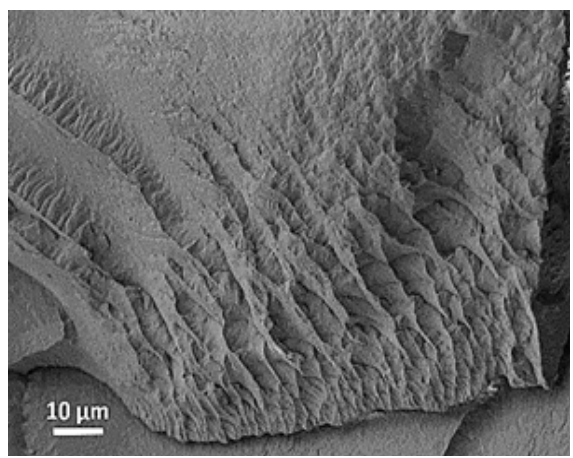

(a)

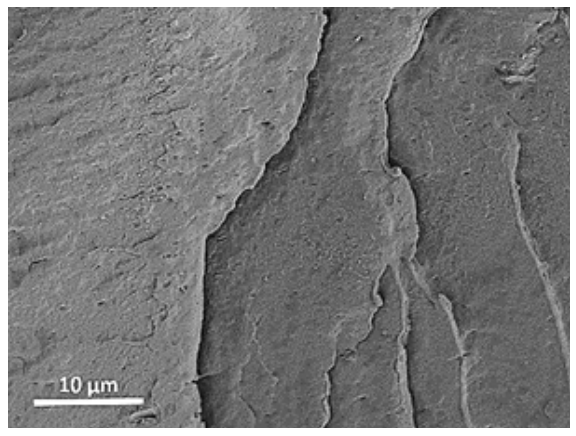

(b)

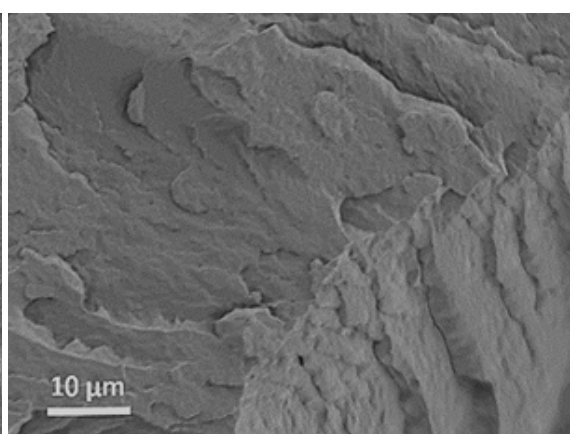

(c)

Figure 11. SEM images of freezed fracture surfaces of (a) neat PA6.6, (b) PA6.6 + 1 wt\% CNF-TEGO, and (c) PA6.6 + 1 wt\% CNF-GO.

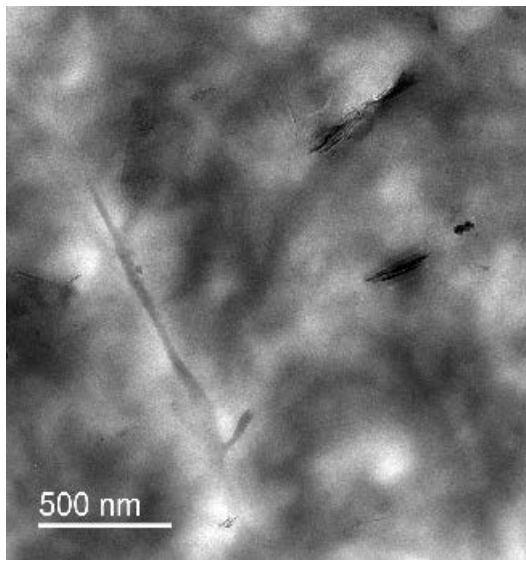

(a)

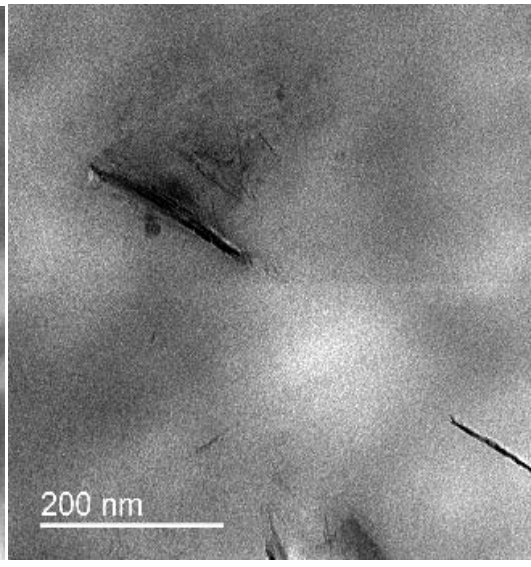

(b) 


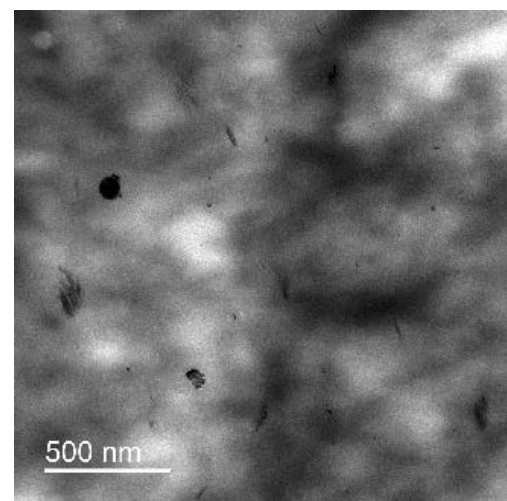

(c)

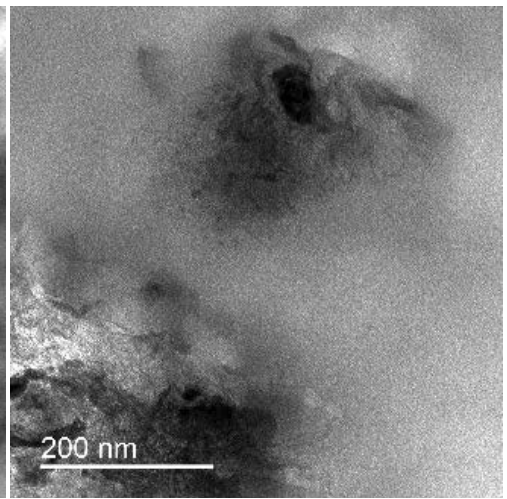

(d)

Figure 12. TEM images of microtome surfaces of (a) and (b) PA6.6 + 1 wt\% CNF-TEGO, and (c) and (d) PA6.6 + 1 wt\% CNF-GO.

\section{Conclusion}

In the current study, hybrid additive having different number of graphene layers and their integration into PA6.6 matrix were investigated in order to understand the interfacial interactions between polymer and graphene based additives and also the behavior of dispersion state. Long CNFs more than $1.5 \mu \mathrm{m}$ were successfully grown on iron loaded single- and multi-layer GO by CVD technique. The structures of these two hybrid additives were confirmed by spectroscopic and macroscopic characterization tools. These CVD grown CNFs on single- and multi-layer GO additives were dispersed into PA6.6 matrix by high shear mixer in melt phase. Tensile test results showed that tensile modulus of nanocomposites was enhanced up to $24 \%$ by the incorporation of $0.5 \mathrm{wt} \%$ CNF-TEGO. The flexural properties of CNF-TEGO reinforced nanocomposites were higher than that of CNF-GO reinforced composites. Especially stronger interfacial interactions were achieved by CNF-TEGO nanoadditive and thus its nanocomposite showed better mechanical performance. This came from the formation of intercalated structure in polymer matrix by preserving the multi-layer nature of TEGO and thus provided higher crystallinity instead of single-layer based hybrid additive. In addition, these new design hybrid additives acted as a reinforcing and nucleating agent in polyamide matrix. Consequently, this study supported that further improvement in graphene structure will influence the performance of structural composites especially used for load bearing applications in a positive manner.

\section{Acknowledgements}

The authors would like to thank Kordsa Teknik Tekstil A.S. Company for providing financial support. This work is also supported by ESTEEM3 project and its grant agreement number is 823717 .

\section{Conflicts of Interest}

The authors declare no conflicts of interest regarding the publication of this paper. 


\section{References}

[1] Bhattacharya, M. (2016) Polymer Nanocomposites-A Comparison between Carbon Nanotubes, Graphene, and Clay as Nanofillers. Materials, 9, 1-35. https://doi.org/10.3390/ma9040262

[2] Edwards, R.S. and Coleman, K.S. (2013) Graphene Synthesis: Relationship to Applications. Nanoscale, 5, 38-51. https://doi.org/10.1039/C2NR32629A

[3] Luo, B., Liu, S. and Zhi, L. (2012) Chemical Approaches toward Graphene-Based Nanomaterials and Their Applications in Energy-Related Areas. Small, 8, 630-646. https://doi.org/10.1002/smll.201101396

[4] Stankovich, S., Dikin, D., Piner, R.D., Kohlhaas, K., Kleinhammes, A., Jia, Y., et al. (2007) Synthesis of Graphene-Based Nanosheets via Chemical Reduction of Exfoliated Graphite Oxide. Carbon, 45, 1558-1565. https://doi.org/10.1016/j.carbon.2007.02.034

[5] Park, S. and Ruoff, R.S. (2009) Chemical Methods for the Production of Graphenes. Nature Nanotechnology, 4, 217-224. https://doi.org/10.1038/nnano.2009.58

[6] Kim, K.S., Zhao, Y., Jang, H., Lee, S.Y., Kim, J.M., Kim, K.S., et al. (2009) Large-Scale Pattern Growth of Graphene Films for Stretchable Transparent Electrodes. Nature, 457, 706-710. https://doi.org/10.1038/nature07719

[7] Marcano, D.C., Kosynkin, D.V., Berlin, J.M., Sinitskii, A., Sun, Z., Slesarev, A., et al. (2010) Improved Synthesis of Graphene Oxide. ACS Nano, 4, 4806-4814. https://doi.org/10.1021/nn1006368

[8] Potts, J.R., Dreyer, D.R., Bielawski, C.W. and Ruoff, R.S. (2011) Graphene-Based Polymer Nanocomposites. Polymer, 52, 5-25. https://doi.org/10.1016/j.polymer.2010.11.042

[9] William, S., Hummers, J. and Offeman, R.E. (1958) Preparation of Graphitic Oxide. Journal of the American Chemical Society, 80, 1339-1339. https://doi.org/10.1021/ja01539a017

[10] Dreyer, D.R., Park, S., Bielawski, C.W. and Ruoff, R.S. (2010) The Chemistry of Graphene Oxide. Chemical Society Reviews, 39, 228-240. https://doi.org/10.1039/B917103G

[11] Saner, B., Okyay, F. and Yürüm, Y. (2010) Utilization of Multiple Graphene Layers in Fuel Cells. 1. An Improved Technique for the Exfoliation of Graphene-Based Nanosheets from Graphite. Fuel, 89, 1903-1910. https://doi.org/10.1016/j.fuel.2010.03.036

[12] Prud'homme, R.K. and Aksay, I.A. (2005) Tire Containing Thermally Exfoliated Graphite Oxide. US Patent No. 8047248B2.

[13] Chen, J., Yao, B., Li, C. and Shi, G. (2013) An Improved Hummers Method for Eco-Friendly Synthesis of Graphene Oxide. Carbon, 64, 225-229. https://doi.org/10.1016/j.carbon.2013.07.055

[14] Fan, Z., Yan, J., Zhi, L., Zhang, Q., Wei, T., Feng, J., et al. (2010) A Three-Dimensional Carbon Nanotube/Graphene Sandwich and Its Application as Electrode in Supercapacitors. Advanced Materials, 22, 3723-3728. https://doi.org/10.1002/adma.201001029

[15] Simari, C., Potsi, G., Policicchio, A., Perrotta, I. and Nicotera, I. (2016) Clay-Carbon Nanotubes Hybrid Materials for Nanocomposite Membranes: Advantages of Branched Structure for Proton Transport under Low Humidity Conditions in PEMFCs. Journal of Physical Chemistry C, 120, 2574-2584.

https://doi.org/10.1021/acs.jpcc.5b11871 
[16] Cheng, H., Dong, Z., Hu, C., Zhao, Y., Hu, Y., Qu, L., et al. (2013) Textile Electrodes Woven by Carbon Nanotube-Graphene Hybrid Fibers for Flexible Electrochemical Capacitors. Nanoscale, 5, 3428-3434. https://doi.org/10.1039/c3nr00320e

[17] Li, W., Dichiara, A. and Bai, J. (2013) Carbon Nanotube-Graphene Nanoplatelet Hybrids as High-Performance Multifunctional Reinforcements in Epoxy Composites. Composites Science and Technology, 74, 221-227. https://doi.org/10.1016/j.compscitech.2012.11.015

[18] Zhou, T., Zha, J.-W., Hou, Y., Wang, D., Zhao, J. and Dang, Z.-M. (2011) Surface-Functionalized MWNTs with Emeraldine Base: Preparation and Improving Dielectric Properties of Polymer Nanocomposites. ACS Applied Materials \& Interfaces, 3, 4557-4560. https://doi.org/10.1021/am201454e

[19] Zhao, T., Palardy, G., Villegas, I.F. and Rans, C. (2017) Mechanical Behaviour of Thermoplastic Composites Spot-Welded and Mechanically Fastened Joints: A Preliminary Comparison. Composites Part B, 112, 224-234. https://doi.org/10.1016/j.compositesb.2016.12.028

[20] Yashas Gowda, T.G.., Sanjay, M.R., Subrahmanya Bhat, K., Madhu, P., Senthamaraikannan, P. and Yohesha, B. (2018) Polymer Matrix-Natural Fiber Composites: An Overview. Cogent Engineering, 5, 1-13. https://doi.org/10.1080/23311916.2018.1446667

[21] Feldman, D. (2017) Polyamide Nanocomposites. Journal of Macromolecular Science, Part A: Pure and Applied Chemistry, 54, 255-262. https://doi.org/10.1080/10601325.2017.1282700

[22] Qiu, L., Yang, Y., Xu, L. and Liu, X. (2013) Influence of Surface Modification of Carbon Nanotube on Microstructures and Properties of Polyamide 66/Multiwalled Carbon Nanotube Composites. Polymer Composites, 34, 656-664. https://doi.org/10.1002/pc.22465

[23] Pan, Y., Hong, N., Zhan, J., Wang, B., Song, L. and Hu, Y. (2014) Effect of Graphene on the Fire and Mechanical Performances of Glass Fiber-Reinforced Polyamide 6 Composites Containing Aluminum Hypophosphite. Polymer-Plastics Technology and Engineering, 53, 1467-1475. https://doi.org/10.1080/03602559.2014.909483

[24] Marcano, D.C., Kosynkin, D.V., Berlin, J.M., Sinitskii, A., Sun, Z., Slesarev, A.S., et al. (2018) Correction to Improved Synthesis of Graphene Oxide. ACS Nano, 12, 2078-2078. https://doi.org/10.1021/acsnano.8b00128

[25] Gohier, A., Ewels, C.P., Minea, T.M. and Djouadi, M.A. (2008) Carbon Nanotube Growth Mechanism Switches from Tip- to Base-Growth with Decreasing Catalyst Particle Size. Carbon, 46, 1331-1338.

[26] SeyyedMonfaredZanjani, J., Saner Okan, B. and Menceloglu, Y. (2016) Manufacturing of Multilayer Graphene Oxide/Poly(Ethylene Terephthalate) Nanocomposites with Tunable Crystallinity, Chain Orientations and Thermal Transitions. Materials Chemistry and Physics, 176, 58-67. https://doi.org/10.1016/j.matchemphys.2016.03.020

[27] Dresselhaus, M.S., Jorio, A., Hofmann, M., Dresselhaus, G. and Saito, R. (2010) Perspectives on Carbon Nanotubes and Graphene Raman Spectroscopy. Nano Letters, 10, 751-758. https://doi.org/10.1021/nl904286r

[28] O’Neill, A., Bakirtzis, D. and Dixon, D. (2014) Polyamide 6/Graphene Composites: The Effect of In Situ Polymerisation on the Structure and Properties of Graphene Oxide and Reduced Graphene Oxide. European Polymer Journal, 59, 353-362. https://doi.org/10.1016/j.eurpolymj.2014.07.038

[29] Kudin, K.N., Ozbas, B., Schniepp, H.C., Prud'homme, R.K., Aksay, I.A. and Car, R. 
(2008) Raman Spectra of Graphite Oxide and Functionalized Graphene Sheets. Nano Letters, 8, 36-41. https://doi.org/10.1021/nl071822y

[30] Grosvenor, A.P., Kobe, B.A., Biesinger, M.C. and McIntyre, N.S. (2004) Investigation of Multiplet Splitting of Fe 2p XPS Spectra and Bonding in Iron Compounds. Surface and Interface Analysis, 36, 1564-1574. https://doi.org/10.1002/sia.1984

[31] Das, R., Hamid, S., Ali, M., Ramakrishna, S. and Yongzhi, W. (2014) Carbon Nanotubes Characterization by X-Ray Powder Diffraction-A Review. Current Nanoscience, 11, 23-35. https://doi.org/10.2174/1573413710666140818210043

[32] Kumar, R. and Sahoo, B. (2018) Carbon Nanotubes or Carbon Globules: Optimization of the Pyrolytic Synthesis Parameters and Study of the Magnetic Properties. Nano-Structures and Nano-Objects, 14, 131-137. https://doi.org/10.1016/j.nanoso.2018.01.014

[33] Zhang, H., Gong, Q., Ren, S., Arshid, M.A., Chu, W. and Chen, C. (2018) Implication of Iron Nitride Species to Enhance the Catalytic Activity and Stability of Carbon Nanotubes Supported Fe Catalysts for Carbon-Free Hydrogen Production via Low-Temperature Ammonia Decomposition. Catalysis Science and Technology, 8, 907-915. https://doi.org/10.1039/C7CY02270K

[34] Liu, J., Yu, B., Zhang, Q., Hou, L., Huang, Q., Song, C., et al. (2015) Synthesis and Magnetic Properties of $\mathrm{Fe}_{3} \mathrm{C}-\mathrm{C}$ Core-Shell Nanoparticles. Nanotechnology, 26, 1-7. https://doi.org/10.1088/0957-4484/26/8/085601

[35] Wang, H., Robinson, J.T., Li, X. and Dai, H. (2009) Solvothermal Reduction of Chemically Exfoliated Graphene Sheets. Journal of the American Chemical Society, 131, 9910-9911. https://doi.org/10.1021/ja904251p

[36] Dresselhaus, M.S., Dresselhaus, G., Saito, R. and Jorio, A. (2005) Raman Spectroscopy of Carbon Nanotubes. Physics Reports, 409, 47-99. https://doi.org/10.1016/j.physrep.2004.10.006

[37] Ferrari, A.C. and Robertson, J. (2000) Interpretation of Raman Spectra of Disordered and Amorphous Carbon. Physical Review B, 61, 14095-14107. https://doi.org/10.1103/PhysRevB.61.14095

[38] Papadopoulou, E.L., Pignatelli, F., Marras, S., Marini, L., Davis, A., Athanassiou, A., et al. (2016) Nylon 6,6/Graphene Nanoplatelet Composite Films Obtained from a New Solvent. RSC Advances, 6, 6823-6831. https://doi.org/10.1039/C5RA23647A

[39] Farias-Aguilar, J.C., Ramírez-Moreno, M.J., Téllez-Jurado, L. and Balmori-Ramírez, H. (2014) Low Pressure and Low Temperature Synthesis of Polyamide-6 (PA6) Using $\mathrm{NaO}$ as Catalyst. Materials Letters, 136, 388-392. https://doi.org/10.1016/j.matlet.2014.08.071

[40] Zhang, G.Z., Yoshida, H. and Kawai, T. (2004) Miscibility of Nylon 66 and Nylon 48 Blend Evaluated by Crystallization Dynamics. Thermochimica Acta, 416, 79-85. https://doi.org/10.1016/j.tca.2003.01.002

[41] Wang, S., Hu, Y., Li, Z., Wang, Z., Zhuang, Y., Chen, Z., et al. (2003) Flammability and Phase-Transition Studies of Nylon 6/Montmorillonite Nanocomposites. Colloid and Polymer Science, 281, 951-956. https://doi.org/10.1007/s00396-002-0858-x

[42] Lu, Y., Zhang, Y., Zhang, G., Yang, M., Yan, S. and Shen, D. (2004) Influence of Thermal Processing on the Perfection of Crystals in Polyamide 66 and Polyamide 66/Clay Nanocomposites. Polymer, 45, 8999-9009. https://doi.org/10.1016/j.polymer.2004.10.025

[43] Lim, L., Britt, I.J. and Tung, M. (1998) Sorption and Transport of Water Vapor in Nylon 6,6 Film. Journal of Applied Polymer Science, 71, 197-206. https://doi.org/10.1002/(SICI)1097-4628(19990110)71:2<197::AID-APP2>3.0.CO;2-J 
[44] Lin, D.J., Chang, C.L., Lee, C.K. and Cheng, L.P. (2006) Fine Structure and Crystallinity of Porous Nylon 66 Membranes Prepared by Phase Inversion in the Water/Formic Acid/Nylon 66 System. European Polymer Journal, 42, 356-367. https://doi.org/10.1016/j.eurpolymj.2005.07.007

[45] Vasanthan, N. and Salem, D.R. (2000) Infrared Spectroscopic Characterization of Oriented Polyamide 66: Band Assignment and Crystallinity Measurement. Journal of Polymer Science, Part B: Polymer Physics, 38, 516-524.

https://doi.org/10.1002/(SICI)1099-0488(20000215)38:4<516::AID-POLB3>3.0.CO; $\underline{2-\mathrm{Y}}$

\section{Nomenclature}

\begin{tabular}{ll}
\hline CVD & Chemical Vapor Deposition \\
\hline PA6.6 & Polyamide 6.6 \\
TEGO & Multi-layer thermally exfoliated graphene oxide \\
CNF & Carbon nanofiber \\
CNF-TEGO & $\begin{array}{l}\text { CVD grown CNF on multi-layer thermally exfoliated } \\
\text { graphene oxide }\end{array}$ \\
GO & Single-layer graphene oxide \\
CNF-GO & CVD grown CNF on single-layer graphene oxide \\
PA6.6+ $(0.25,0.5,1) \mathrm{wt} \%$ TEGO & $(0.25,0.5,1) \mathrm{wt} \%$ TEGO loaded PA6.6 nanocomposite \\
PA6.6 + $(0.25,0.5,1) \mathrm{wt} \%$ CNF-TEGO & $\begin{array}{l}(0.25,0.5,1) \mathrm{wt} \% \text { CNF-TEGO loaded PA6.6 } \\
\text { nanocomposite }\end{array}$ \\
PA6.6 + $(0.25,0.5,1) \mathrm{wt} \%$ GO & $(0.25,0.5,1) \mathrm{wt} \%$ GO loaded PA6.6 nanocomposite \\
PA6.6 + $(0.25,0.5,1) \mathrm{wt} \%$ CNF-GO & $(0.25,0.5,1) \mathrm{wt} \%$ CNF-GO loaded PA6.6 nanocomposite
\end{tabular}




\section{Supplementary Data}

Table S1. XPS results of neat GO samples and their hybrid additives.

\begin{tabular}{ccccc}
\hline Samples & C (at\%) & O (at\%) & Other (at\%) & C/O \\
\hline GO & 60.59 & 34.28 & 5.13 & 0.5 \\
CNF-GO & 96.83 & 3.17 & - & 5.5 \\
TEGO & 97.94 & 2.06 & - & 3.4 \\
CNF-TEGO & 98.12 & 1.88 & - & 6.9 \\
\hline
\end{tabular}

Table S2. XPS spectra results for C1s, O1s, and Fe2p in the CNF-TEGO and CNF-GO [1] [2] [3] [4] [5].

\begin{tabular}{|c|c|c|c|c|c|c|c|c|c|}
\hline \multirow[b]{3}{*}{ Samples } & \multicolumn{3}{|c|}{ C1s } & \multicolumn{3}{|c|}{ O1s } & \multicolumn{3}{|c|}{$\mathrm{Fe} 2 \mathrm{p}$} \\
\hline & & Binding & Peak & & Binding & Peak & & Binding & Peak \\
\hline & Group & $\begin{array}{c}\text { Energy } \\
(\mathrm{eV})\end{array}$ & $\begin{array}{c}\text { Intensity } \\
\text { (a.u.) }\end{array}$ & Group & $\begin{array}{c}\text { Energy } \\
(\mathrm{eV})\end{array}$ & $\begin{array}{c}\text { Intensity } \\
\text { (a.u.) }\end{array}$ & Group & $\begin{array}{c}\text { Energy } \\
(\mathrm{eV})\end{array}$ & $\begin{array}{c}\text { Intensity } \\
\text { (a.u.) }\end{array}$ \\
\hline \multirow{6}{*}{ TEGO } & $\mathrm{C}=\mathrm{C}$ & 284.1 & 104,164 & $\mathrm{C}-\mathrm{O}$ & 530.6 & 10,588 & & & \\
\hline & $\mathrm{C}-\mathrm{C}$ & 284.8 & 7664 & $\mathrm{C}=\mathrm{O}$ & 532.5 & 12,820 & & & \\
\hline & $\mathrm{C}-\mathrm{O}$ & 286.3 & 7339 & $\mathrm{HO}-\mathrm{C}=\mathrm{O}$ & 535.6 & 11,334 & & & \\
\hline & $\mathrm{O}-\mathrm{C}=\mathrm{O}$ & 288.9 & 7858 & & & & & & \\
\hline & $\begin{array}{l}\mathrm{C}=\mathrm{C}, \\
\mathrm{C}-\mathrm{Fe}^{*}\end{array}$ & 284.3 & 510,259 & $\mathrm{C}-\mathrm{O}, \mathrm{Fe}-\mathrm{O}$ & 529.8 & 38,318 & $\mathrm{Fe} 2 \mathrm{p} 3 / 2$ & 710.6 & 32,860 \\
\hline & $\mathrm{C}-\mathrm{C}$ & 285.2 & 76,341 & $\mathrm{C}=\mathrm{O}$ & 532 & 45,209 & Satellite & 719.5 & 31,530 \\
\hline \multirow[t]{3}{*}{ CNF-TEGO } & $\mathrm{C}-\mathrm{O}-\mathrm{C}$ & 287.2 & 50,557 & & & & $\mathrm{Fe} 2 \mathrm{p} 1 / 2$ & 724.2 & 32,214 \\
\hline & $\mathrm{O}-\mathrm{C}=\mathrm{O}$ & 290.7 & 45,214 & & & & Satellite & 727.6 & 31,657 \\
\hline & $\mathrm{C}-\mathrm{C}$ & 284.7 & 51,245 & $\mathrm{C}-\mathrm{O}$ & 531.8 & 54,857 & & & \\
\hline \multirow[t]{4}{*}{$\mathrm{GO}$} & $\mathrm{C}-\mathrm{O}$ & 286.9 & 66,274 & $\mathrm{C}=\mathrm{O}$ & 532.7 & 80,534 & & & \\
\hline & $\mathrm{O}-\mathrm{C}=\mathrm{O}$ & 288.7 & 19,284 & $\mathrm{HO}-\mathrm{C}=\mathrm{O}$ & 533.3 & 82,456 & & & \\
\hline & $\begin{array}{l}\mathrm{C}=\mathrm{C}, \\
\mathrm{C}-\mathrm{Fe}^{*}\end{array}$ & 284.3 & 278,137 & $\mathrm{C}-\mathrm{O}, \mathrm{Fe}-\mathrm{O}$ & 529.9 & 27,211 & $\mathrm{Fe} 2 \mathrm{p} 3 / 2$ & 710.7 & 37,089 \\
\hline & $\mathrm{C}-\mathrm{C}$ & 285.4 & 46,628 & $\mathrm{C}=\mathrm{O}$ & 532.1 & 31,673 & Satellite & 720.0 & 36,416 \\
\hline \multirow[t]{2}{*}{ CNF-GO } & $\mathrm{C}-\mathrm{O}-\mathrm{C}$ & 287.1 & 37,166 & $\mathrm{HO}-\mathrm{C}=\mathrm{O}$ & 533.4 & 27,484 & $\mathrm{Fe} 2 \mathrm{p} 1 / 2$ & 724.5 & 36,759 \\
\hline & $\mathrm{O}-\mathrm{C}=\mathrm{O}$ & 290.5 & 31,652 & & & & Satellite & 727.7 & 36,284 \\
\hline
\end{tabular}

Table S3. Raman peak positions, intensities and $\mathrm{I}_{\mathrm{D}} / \mathrm{I}_{\mathrm{G}}$ values of TEGO and GO and their hybrids with CNF.

\begin{tabular}{ccccc}
\hline & $\mathrm{D}$ & $\mathrm{G}$ & $2 \mathrm{D}$ & $\mathrm{I}_{\mathrm{D}} / \mathrm{I}_{\mathrm{G}}$ \\
\cline { 2 - 5 } & $\begin{array}{c}\text { Position }\left(\mathrm{cm}^{-1}\right) / \\
\text { Intensity }(\mathrm{au})\end{array}$ & $\begin{array}{c}\text { Position }\left(\mathrm{cm}^{-1}\right) / \\
\text { Intensity }(\mathrm{au})\end{array}$ & $\begin{array}{c}\text { Position }\left(\mathrm{cm}^{-1}\right) / \\
\text { Intensity }(\mathrm{au})\end{array}$ & \\
\hline \multirow{2}{*}{ TEGO } & - & $1582 /$ & $2721 /$ & - \\
& & 6728 & 4309 & \\
CNF-TEGO & $1342 /$ & $1579 /$ & $2689 /$ & \multirow{2}{*}{1.5} \\
& 6399 & 4277 & 2203 & \\
\multirow{2}{*}{ GO } & $1355 /$ & $1593 /$ & - & \\
& 14809 & 15835 & - & 1.5 \\
\multirow{2}{*}{ CNF-GO } & $1349 /$ & $1571 /$ & & \\
& 5445 & 3503 & &
\end{tabular}


Table S4. The transmittance ratio of crystalline and independent peak $\left(\mathrm{T}_{1198 / 1630}\right)$.

\begin{tabular}{cccc}
\hline Samples & $\mathrm{T}_{1198}$ & $\mathrm{~T}_{1630}$ & $\mathrm{~T}_{1198 / 1630}$ \\
\hline Neat PA6.6 & 86.6 & 72.4 & 1.20 \\
PA6.6 + 0.25 wt\% CNF-TEGO & 74.9 & 42.0 & 1.78 \\
PA6.6 + 0.5 wt\% CNF-TEGO & 72.4 & 36.3 & 1.99 \\
PA6.6 + 1 wt\% CNF-TEGO & 80.5 & 55.3 & 1.46 \\
PA6.6 + 0.25 wt\% CNF-GO & 77.5 & 33.7 & 2.30 \\
PA6.6 + 0.5 wt\% CNF-GO & 76.5 & 29.5 & 2.60 \\
PA6.6 + 1 wt\% CNF-GO & 72.2 & 44.6 & 1.62
\end{tabular}

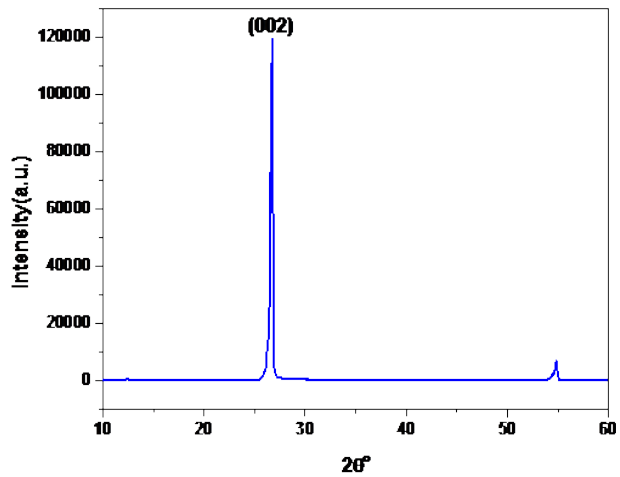

(a)

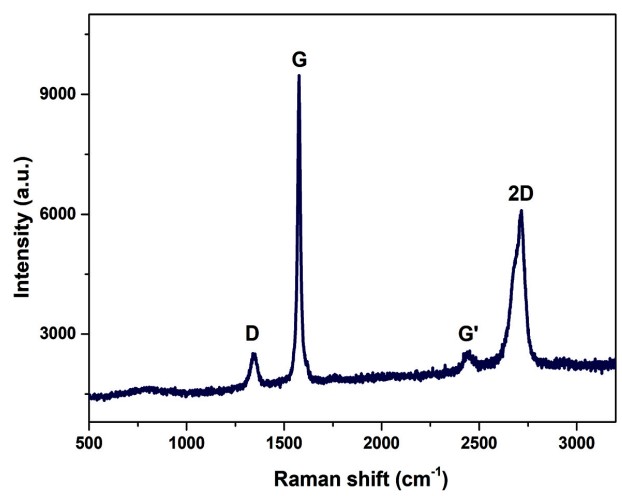

(b)

Figure S1. (a) XRD spectrum and (b) Raman spectrum of graphite flake.

\section{References (Supplementary Data)}

[1] Che, J., Shen, L. and Xiao, Y. (2010) A New Approach to Fabricate Graphene Nanosheets in Organic Medium: Combination of Reduction and Dispersion. Journal of Materials Chemistry, 20, 1722-1727. https://doi.org/10.1039/b922667b

[2] Grosvenor, A.P., Kobe, B.A., Biesinger, M.C. and McIntyre, N.S. (2004) Investigation of Multiplet Splitting of Fe 2p XPS Spectra and Bonding in Iron Compounds. Surface and Interface Analysis, 36, 1564-1574. https://doi.org/10.1002/sia.1984

[3] Hallam, P.M., Gómez-Mingot, M., Kampouris, D.K. and Banks, C.E. (2012) Facile Synthetic Fabrication of Iron Oxide Particles and Novel Hydrogen Superoxide Su- 
percapacitors. RSC Advances, 2, 6672-6679. https://doi.org/10.1039/c2ra01139e

[4] Yamashita, T. and Hayes, P. (2008) Analysis of XPS Spectra of $\mathrm{Fe}^{2+}$ and $\mathrm{Fe}^{3+}$ Ions in Oxide Materials. Applied Surface Science, 254, 2441-2449.

https://doi.org/10.1016/j.apsusc.2007.09.063

[5] Okan, B.S., Yürüm, A., Gorgülü, N., Gürsel, S.A. and Yürüm, Y. (2011) Polypyrrole Coated Thermally Exfoliated Graphite Nanoplatelets and the Effect of Oxygen Surface Groups on the Interaction of Platinum Catalysts with Graphene-Based Nanocomposites. Industrial and Engineering Chemistry Research, 50, 12562-12571. https://doi.org/10.1021/ie201613f 